\title{
EL TALler de LENGUAJE Y COMUNiCACión COMO VÍA PARA MEJORAR LA ENSEÑANZA-APRENDIZAJE DE ORTOGRAFÍA Y COMPRENSIÓN LECTORA EN ESTUDIANTES UNIVERSITARIOS
}

\author{
THE LANGUAGE AND COMMUNICATION WORKSHOP AS A WAY \\ TO IMPROVE THE TEACHING AND LEARNING OF SPELLING AND READING \\ COMPREHENSION IN COLLEGE STUDENTS
}

Mg. Marcela Córdova Alulema
Universidad Israel - Ecuador
mcordova@uisrael.edu.ec
Fecha de recepción: 09/02/2015
Fecha de aceptación: 16/03/2015

Resumen

Todo ser humano tiene la necesidad de comunicarse y de relacionarse con los demás, ya sea en su vida personal o profesional, tomando en cuenta que el proceso de comunicación es probablemente la actividad que más influye en el comportamiento humano. No obstante, estudios realizados al respecto han demostrado problemas en la ortografía y comprensión lectora de los estudiantes universitarios ecuatorianos, estos interfieren en su formación y desempeño profesional; a ello se suman deficiencias metodológicas en la enseñanza aprendizaje en el área de conocimiento que en las diferentes carreras tributa a tales fines como comunicación y lenguaje.

Una de las vías para mejorar la enseñanza del aprendizaje de ortografía y comprensión lectora lo constituye el Taller de Lenguaje y Comunicación por su carácter integrador. A partir de los supuestos anteriores, el presente 
trabajo tiene como finalidad fundamentar teóricamente el taller como vía para mejorar el proceso de enseñanza-aprendizaje de ortografía y comprensión lectora.

Palabras clave: Taller, Enseñanza-Aprendizaje, Ortografía, Comprensión Lectora, Universidad

\begin{abstract}
Every human being has the need to communicate and interact with others, whether it is in your personal or professional life taking into account that the process of communication is probably the activity that most influences human behavior. However, studies on the subject have shown problems in spelling and reading comprehension of Ecuadorian University students that interfere with their training and professional development; this adds methodological deficiencies in the teaching and learning in the area of knowledge which taxed to such purposes as communication and language in the different races.

One of the ways to improve the teaching and learning of spelling and reading comprehension is language and communication workshop for its integrator nature. From the previous assumptions, this paper aims to explain theoretically the workshop as a way to improve the process of teaching - learning of spelling and reading comprehension.
\end{abstract}

Keywords: Workshop, Teaching and Learning, Spelling, Reading Comprehension, University 


\section{Introducción}

En el Ecuador, la educación a nivel de primaria, secundaria y bachillerato ha sido históricamente deficiente, caracterizada, entre otros, por la persistencia del analfabetismo, bajo nivel de escolaridad, tasas elevadas de repetición y deserción escolar, mala calidad de la educación, deficiente infraestructura educativa y limitado material didáctico.

La inversión más importante en el progreso de un país es la educación y son los educadores los que deben tomar en cuenta que los contenidos que se imparten en la escuela, colegio y universidad son secuenciales, lo que requiere de una alta sistematicidad en su enseñanza y comprensión. No es posible aspirar a que el estudiante interiorice una determinada temática, si no ha comprendido toda la materia procedente de años anteriores, por lo que resulta de suma importancia que los estudiantes y docentes asuman con entera responsabilidad el fomentar un adecuado desarrollo de cada una de las asignaturas genéricas.

En los educandos se observan grandes falencias que han ido arrastrando año tras año, entre ellas la deficiente ortografía, comprensión lectora y expresión oral, lo que refleja la condición en la que se encuentran estos.

El ignorar cómo estructurar adecuadamente oraciones, frases, párrafos, entre otros, perjudica a los estudiantes tanto en su vida personal como profesional.

En la Universidad, el área o departamento de Formación Humanística es la que dicta las asignaturas relacionadas con Lenguaje y Comunicación, Lectura Comprensiva, entre otras importantes en la formación de un profesional. Estas asignaturas se imparten aplicando contenidos acordes con las necesidades de desarrollo de capacidades del educando y mediante la utilización de sílabos con una carga horaria de dos a tres horas por semana, horas que resultan insuficientes para cubrir con las múltiples carencias que poseen los estudiantes.

Es por esto que el presente trabajo plantea la posibilidad de implementar talleres de trabajo que ayuden al estudiante a desarrollar su creatividad, habilidad y destreza lingüística. El taller guía al estudiante para que pueda afianzar el aprendizaje de ortografía, comprensión lectora, enriquecimiento del vocabulario, desarrollando conciencia ortográfica permanente y para mejorar la capacidad lingüística. 


\section{Fundamentos para la propuesta del taller como vía para mejorar la enseñanza- aprendizaje de ortografía y comprensión lectora en estudiantes universitarios}

\section{Fundamentos pedagógicos y andragógicos}

Según Álvarez (1999), la Pedagogía es la ciencia que tiene como objeto de estudio el proceso formativo, conceptualizándolo como "un proceso totalizador cuyo objetivo es preparar al hombre como ser social". Para él, el proceso formativo incluye en una unidad dialéctica los procesos educativo, desarrollador e instructivo. Actualmente se considera que la educación no es solo cuestión de niños y adolescentes, lo educativo es un proceso que actúa sobre el hombre a lo largo de toda su vida; la naturaleza del hombre indica que puede continuar aprendiendo durante toda su vida sin importar su edad cronológica.

Al hablar de estudiantes universitarios, se está tratando con personas de edades comprendidas a partir de los 17 años, y si se habla de enseñanza-aprendizaje, tratar de andragogía es lo correcto, ya que como disciplina se ocupa de la educación y el aprendizaje del adulto.

Yturralde (2012) sitúa las particularidades de los procesos de aprendizaje en los adultos, estos con mayor o menor intensidad buscan el conocimiento para su inmediata aplicación práctica que les permita generar cambios o mejoras en sus actividades, tareas, oficios o profesiones.

La Andragogía y la Pedagogía son disciplinas semejantes en ciertos aspectos, como por ejemplo: las dos persiguen un mismo fin, que es el enseñar independientemente de la edad.

La participación activa está determinada por factores como la experiencia, las expectativas, los intereses y las necesidades que el estudiante posea. En tal sentido, le corresponde a los docentes utilizar modelos de aprendizaje andragógicos pertinentes con las edades, características individuales y de grupo, el entorno y la comunidad en general; porque en la praxis andragógica se adquiere una dimensión operativa a través de acciones planificadas, formativas, evaluativas y constructivas para propiciar un adecuado clima de enseñanza.

\section{Aprendizaje Experiencial}

El estudiante universitario posee muchos conocimientos adquiridos en la práctica de sus quehaceres diarios y de acuerdo al entorno en el que se desarrolla, es por esto que para Dewey (1938) toda auténtica educación se efectúa mediante la experiencia.

Él considera que el Aprendizaje Experiencial es activo y genera cambios en las personas y en sus entornos, y que no sólo va al interior del cuerpo y del alma del que aprende, sino que utiliza y transforma los ambientes físicos y sociales. 


\section{Fundamentos psicosociales}

"La tarea del escritor consiste en mostrar cómo el contexto social influye en la psicología personal."

Wolfe, Thomas C.

Los adultos son un cúmulo de experiencias que se convierten en importantes recursos de aprendizaje, son un puente para desarrollar nuevos aprendizajes para sí mismo, así como para la comunidad. El adulto está dispuesto a aprender lo que necesita saber o saber hacer, para así cumplir en la sociedad: laboralmente como profesionales, líderes, trabajadores; familiarmente, como esposos(as), padres o madres y socialmente como vecino, compañero, entre otros. Los adultos están más motivados para aprender por los factores internos, tales como el desarrollo de la autoestima, aunque eventualmente se puede encontrar personas que buscan evitar participar en los procesos de aprendizajes por varios factores, entre estos, el temor a hablar en público, desconocimiento, vergüenza, falta de seguridad.

El educando suele hacer más por evitar sus mayores miedos que lo que hace por alcanzar sus anhelos, pero es parte de una realidad.

\section{Fundamentos lingüísticos y psicolingüísticos}

\section{Fundamentos linguiísticos}

El lenguaje humano se expresa en la capacidad de los seres humanos para comunicarse por medio de signos, principalmente utilizando el signo lingüístico. Este es un sistema de comunicación más especializado que los de otras especies animales, a la vez es fisiológico y psíquico, pertenece tanto al dominio individual como al social y permite abstraer, conceptualizar, y comunicar.

\section{Competencia lingüística}

La lingüística moderna surge a partir del siglo XIX. Con la publicación póstuma del "Curso de lingüística general" de Ferdinand de Saussure (1916), la lingüística se ha convertido en una ciencia integrada a la semiología. Desde ese entonces, aparece la necesidad de marcar una diferencia entre la lengua (entendida como todo el sistema) y el habla (es decir, su puesta en práctica), así como también de rever la definición de signo lingüístico (recurso donde se agrupa al significado y al significante).

Para Tobón (2006) la competencia es un enfoque para la educación y no un modelo pedagógico, y la competencia comunicativa en la educación se orienta al dominio expresivo y comprensivo de los usos verbales y no verbales de la comunicación humana, y para mejorar el aprendizaje de las destrezas del hablar, escuchar, leer, entender y escribir. Porque son estas las habilidades comunicativas que se aprenden en la sociedad para participar de una manera coherente, eficaz y adecuada en los intercambios verbales que caracterizan la comunicación. 
Según los fines de este trabajo se particularizará en dos de los saberes:

Saber escribir: Para Ruiz (2007) saber escribir es una habilidad que permite expresar de una forma más íntima y personalizada la relación afectiva con el contenido de la obra donde están expresos los valores; así como el grado de elaboración personal. Cuando se expresan por escrito: juicios, valoraciones, comentarios, arriban a ellos tras un proceso de análisis, reflexión y valoración expresivos en gran medida de una interiorización manifiesta a través de una verbalización, a diferencia de la lengua oral que es compulsada al calor del grupo. Sin embargo, en la redacción el individuo se expresa por sí solo, de ahí que deban ser actividades de cierre. Ella sugiere que para trabajar la habilidad de escribir se tendrá en cuenta que:

- Deben ser actividades de cierre.

- En un momento en que se haya producido un estado afectivo-motivacional favorable, provocado por las actividades orales.

- Los ejercicios deben ser de composición, pues tienen más libertad expresiva y formal para expresar abiertamente impresiones, juicios, comentarios, valoraciones, etc.

- Es importante realizar un trabajo de socialización grupal de las redacciones realizadas y estimular la creatividad.

Saber leer: Solé (1998) considera la lectura como un objeto de conocimiento en sí mismo y como instrumento necesario para la realización de nuevos aprendizajes.

La autora ha señalado que leer es:

- Un proceso de interacción entre el lector y el texto, proceso mediante el cual el primero intenta satisfacer los objetivos que guían su lectura.

- El proceso mediante el cual se comprende el lenguaje escrito.

El primer aspecto implica la presencia de un lector activo que procesa y examina el texto con el propósito de lograr un objetivo. Por eso dice Cassany (2009) que, en definitiva, la lectura se convierte en un aprendizaje trascendental en aspectos como el éxito o el fracaso educativo, la preparación técnica para acceder al mundo del trabajo, el grado de autonomía y desenvoltura personales, etc. Estas se relacionan directamente con las capacidades de la lectura, y la comprensión lectora como tal y como se concibe actualmente, es un proceso a través del cual el lector elabora un significado en su interacción con el texto (Anderson y Pearson, 1984).

La comprensión a la que el lector llega durante la lectura se deriva de sus experiencias acumuladas y el contexto, experiencias que entran en juego a medida que decodifica las palabras, frases, párrafos e ideas del autor.

La interacción entre el lector y el texto es el fundamento de la comprensión. En este proceso de comprender, el lector relaciona la información que el autor le presenta con la información almacenada en su mente. Este proceso de relacionar la información nueva con la antigua es el proceso de la comprensión. 


\section{Fundamentos psicolingüísticos}

La psicolingüística es una rama de la psicología interesada en cómo la especie humana adquiere y utiliza el lenguaje. Para ello estudia los factores psicológicos, neurológicos que capacitan a los humanos para la adquisición y deterioro del mismo, uso, comprensión del lenguaje y sus funciones cognitivas y comunicativas.

León (2004) precisa acertadamente que la comprensión de lo que se lee depende de procesos psicolingüísticos individuales y personales a través de los cuales el lector construye su propio significado, interactuando con el texto al aportar conocimientos previos y hacer inferencias que guían su comprensión.

Siguiendo al referido autor, se debe expresar que estos procesos son internos y le permiten al lector avanzar y retroceder, recorrer, detenerse, pensar y comprender, y así ir cimentando ideas sobre el contenido del texto porque, en definitiva, la comprensión depende de cada lector y de su forma de interactuar con el texto.

Igualmente enfatiza en que la comprensión se identifica con las formas de entablar relaciones inteligentes entre lo que comprende y el objeto de comprensión y como resultado de una interacción entre varios factores: los procesos cognitivos que afectan a la comprensión misma, esto es, las operaciones mentales que se llevan a cabo, los conocimientos de los que se dispone.

Para Hallyday (1976), el término "texto" se utiliza en lingüística para referirse a un pasaje oral o escrito, no importa de qué extensión, que constituye un todo unificado, bien pudiendo desarrollar la lectura y escritura mediante el uso de un taller de trabajo que permita desarrollar los aspectos psicolingüísticos que se han analizado.

\section{El Taller como vía para mejorar la enseñanza-aprendizaje de ortografía y comprensión lectora en estudiantes universitarios}

El taller como sistema de enseñanza-aprendizaje es una de las llamadas metodologías activas, al respecto nos dice Egg (1999) que el taller en el acto pedagógico está centrado en el grupo que debe realizar un trabajo y este constituye la situación de aprendizaje.

El acto de enseñar es principalmente del docente y la responsabilidad de aprender debe asumirla cada estudiante y para que el estudiante aprenda verdaderamente, debe poder expresarse libremente y razonar sin estar limitado por la disciplina o autoridad formal del docente.

En el campo de la educación, se habla de talleres para nombrar a una cierta metodología de enseñanza que combina la teoría y la práctica. Los talleres permiten el desarrollo de investigaciones y el trabajo en equipo; algunos talleres son permanentes dentro de un cierto nivel educativo, mientras que otros pueden durar uno o varios días y no estar vinculados a un sistema específico. 


\section{El Taller en el proceso de enseñanza aprendizaje}

El trabajo en talleres, asumido por los docentes como un recurso y un procedimiento dirigido a mostrar un compendio de acciones prácticas para la enseñanza y el aprendizaje, es en la actualidad una manifestación del desempeño pedagógico, académico, dentro de las aulas de clases. Ello obedece a la necesidad de proporcionarle al colectivo que aprende un ámbito donde se ofrezca la oportunidad de indagar, averiguar, explorar, experimentar y descubrir un buen número de actividades significativas que indiquen el camino a seguir para lograr la activación del pensamiento y el desarrollo de competencias y habilidades.

\section{Características de un taller}

Egg (1999) nos da a conocer ocho características importantes de un taller de trabajo.

1. Es un aprender haciendo.

2. Es una metodología participativa.

3. Es una pedagogía de la pregunta, contrapuesta a la pedagogía de la repuesta, propia de la pedagogía tradicional.

4. Es un entrenamiento que tiende al trabajo interdisciplinario, y al enfoque sistémico.

5. La relación docente/estudiante queda establecida en la realización de una tarea común.

6. Carácter globalizante e integrador de su práctica pedagógica.

7. Implica y exige de un trabajo grupal, y el uso de técnicas adecuadas

8. Permite integrar un solo proceso tres instancias como son la docencia, la investigación y la práctica

\section{Los tres momentos de un taller}

Cano (2012) propone establecer momentos de ejecución de un taller desde el inicio, la ejecución y la evaluación.

a) Planificación: consiste en tener en cuenta los objetivos que se pretende alcanzar: el para qué de la realización del taller. Las características que adopte el taller, su duración, contenidos, las técnicas que se utilizarán, así como la estrategia de difusión, registro y evaluación, serán diferentes según se trate de un taller de formación, de diagnóstico, de planificación o de evaluación.

b) Desarrollo: se refiere a lo que sucede efectivamente en el taller, con lo que planificamos previamente.

El desarrollo del taller tiene a su vez tres momentos:

APERTURA: si los integrantes del taller no se conocen, es bueno comenzar dedicando un tiempo a la presentación, utilizando eventualmente técnicas específicas para ello. 
DESARROLLO: el desarrollo con flexibilidad y creatividad de las tareas planificadas.

CIERRE: El cierre de la actividad es un momento importante en sí mismo, y además es el momento en que se realiza la evaluación del taller.

c) Evaluación: refiere al análisis y reflexión de lo producido en el taller.La consideración de lo que vimos, pensamos y sentimos en torno a lo que sucedió en el taller. La evaluación abarca tanto lo producido-creado en el taller, como los aspectos referentes a cómo nos sentimos durante el mismo.

\section{Componentes de un taller}

En función de los momentos de un taller expresados por Cano (2010), la autora propone el siguiente esquema de trabajo para los componentes de un taller:

- Presentación: proporciona información necesaria para que se pueda desarrollar el taller, al final de la lectura el estudiante estará en la capacidad de discernir lo que va hacer.

- Responsable: se encarga de organizar la preparación y realización, determinando las actividades que se llevarán a cabo en los talleres. Es guía para los participantes y se observa la dinámica del grupo para crear una atmósfera adecuada para el desarrollo del taller.

- Objetivos: son el resultado que se espera si el estudiante finaliza determinado aprendizaje, estas actividades pueden convertirse en un parámetro de evaluación.

- Recursos didácticos: son un conjunto de materiales que se han elaborado para facilitar la enseñanzaaprendizaje, ayudan a facilitar las habilidades de los estudiantes y también a desarrollarlas, contribuyen a maximizar la motivación.

- Actividades: conjunto de acciones que se llevan a cabo para cumplir con metas u objetivos de determinado programa. Consiste en la ejecución de ciertos procesos o tareas que pueden ser ayudados por el uso de recursos didácticos.

- Evaluación: es un proceso que tiene como finalidad determinar el grado de eficacia y eficiencia con que han sido empleados los recursos destinados para alcanzar los objetivos previstos.

Posibilita la determinación de las desviaciones y la adopción de medidas correctivas que garanticen el cumplimiento adecuado de las metas presupuestadas. Se aplica ex ante (antes de), concomitante (durante), y ex post (después de) de las actividades desarrolladas. 


\section{El taller de lenguaje y comunicación para mejorar la enseñanza-aprendizaje de ortografía y comprensión lectora}

Egg (1999) manifiesta que el taller es un lugar donde se trabaja, se elabora y se transforma algo para ser utilizado y desde el punto de vista de la lecto-escritura sirve para:

- Escribir bien.

- Adecuar la escritura al contexto y a la situación particular.

- Tener en cuenta que, a pesar de lo anterior, existe una normativa que hay que seguir.

Los talleres de escritura pueden diferir en la metodología o en la forma de desarrollar la escritura; pero tienen la misma filosofía y un objetivo común: crear un espacio (real o virtual) en el que las personas puedan mejorarla a través de la única manera que se puede, es decir, escribiendo.

Es una experiencia de un trabajo concreto. Su punto final debe ser un compromiso de ejecutar acciones, en tareas realizables a corto y mediano plazo.

A continuación se presentan talleres prácticos en los que el estudiante va a poner en juego su razonamiento lógico, destrezas y habilidades, las que pueden ser desarrolladas de forma individual o grupal con o sin ayuda del docente, ya que contiene al final las repuestas de cada taller para que el educando pueda verificar su avance.

\section{Propuesta de talleres Presentación}

El presente taller se propone para utilizarse en nivelaciones, propedéuticos, nivel inicial superior, en sistemas escolarizados, abiertos o por medio de métodos de autoaprendizaje, y por supuesto, al público en general.

Debido a la flexibilidad, organización y conformación de la obra, el estudiante podrá abordar todos los temas sin necesidad de apoyarse de un especialista en la materia.

Las estrategias de aprendizaje empleadas son variadas, como: deducir e inducir ciertas reglas, subrayar, realizar ejercicios de memorización visual; observar, repasar, ejercitar y aplicar distintas normas ortográficas. También se han incorporado ejercicios de lectura comprensiva para que el estudiante compruebe su mejora en el aprendizaje; se ha incluido una sección de respuestas a los ejercicios propuestos.

Estos talleres fueron elaborados para desarrollar en el estudiante destrezas, habilidades, actitudes y conocimientos dentro del contexto de la ortografía y la lectura comprensiva, aspecto fundamental para el mejor desempeño en cualquier asignatura. 
Los ejercicios y los textos, por su parte, promueven la sensibilidad por el arte y el aprendizaje por iniciativa e interés propio, la vinculación de la ortografía con la vida cotidiana y la transversalidad con otras materias. Además, los diferentes ejercicios invitan a aprender a pensar, deducir, reflexionar, discriminar y concluir.

La propuesta metodológica del taller de trabajo de lenguaje y comunicación permite fortalecer los hábitos en el estudiante, relacionados con la experiencia de la práctica ortográfica y la frecuente lectura.

\section{Objetivo general}

Mejorar el aprendizaje de ortografía, comprensión lectora y el enriquecimiento del vocabulario, desarrollando conciencia ortográfica permanente para incorporarla como herramienta que fortalezca la calidad educativa.

\section{Características}

El taller está constituido por actividades con la siguiente estructura:

Nombre: contiene el nombre de cada taller.

Objetivo: determina lo que se quiere alcanzar en cada taller.

Orientación didáctica: precisa las actividades que se van a realizar.

Recursos: indica los materiales a utilizar en cada taller.

Evaluación: verifica el correcto desarrollo de los ejercicios realizados.

Sugerencias: son indicaciones que pueden ayudar a desarrollar las tareas.

\section{Contenidos}

Taller uno: Lectura Estrategias de comprensión lectora

Taller dos: Ejercicios de lectura comprensiva

Taller tres: Ortografía Ejercicios con palabras homófonas, vocabulario.

Taller cuatro: Ejercicios con antónimos - si- nónimos, ortografía, estructura de oraciones

Taller cinco: Ejercicios de lectura y aplicación ortográfica. 


\section{Estructura de la propuesta de talleres}

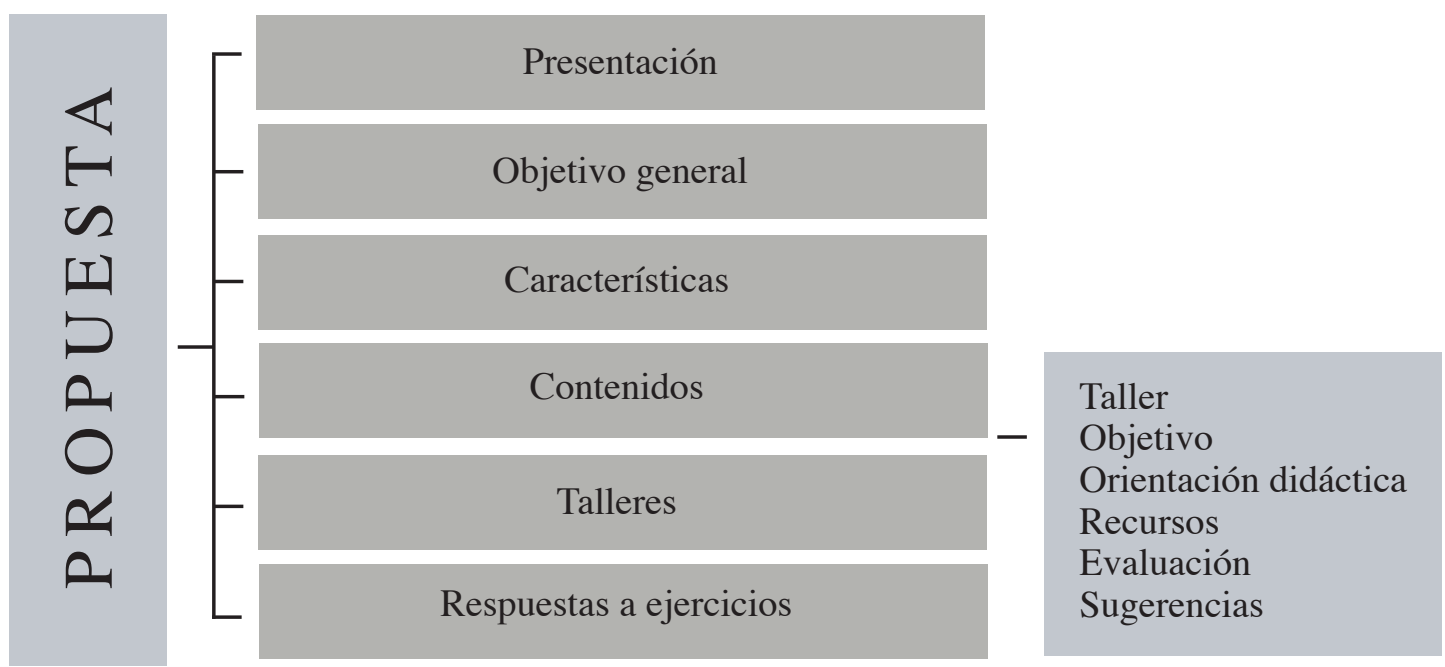

\section{Sugerencias para aplicar los talleres}

El docente, como actor directo en el proceso de enseñanza-aprendizaje debe:

- Planificar con anterioridad el uso del taller.

- Determinar un horario permanente para ejecutar los ejercicios.

- Revisar los ejercicios a ejecutar y tener a mano un diccionario.

- Motivar y concienciar al estudiantado de la importancia de cada clase.

- Dar a conocer el objetivo de cada taller.

- Iniciar con los ejercicios más fáciles para luego realizar los más complejos.

- Determinar un tiempo estimado para cada grupo de ejercicios.

\section{Orientaciones metodológicas}

La metodología a aplicarse incluye la práctica de diferentes tipos de ejercicios que pueden ser desarrollados de la siguiente manera:

- Vivenciales (de animación o de análisis). 
- Con actuación (por ejemplo juego de roles, dramatización de cuentos).

- Auditivas y audiovisuales (ejemplo radio o video foro).

- Visuales (escritas y gráficas).

\section{Propuesta de evaluación}

Tomando en cuenta que la evaluación se puede concebir como "una fase de control que tiene como objeto no sólo la revisión de lo realizado sino también el análisis so- bre las causas y razones para determinados resultados, y la elaboración de un nuevo plan en la medida que proporciona antece- dentes para el diagnóstico" (Duque, 1993: p.167), la autora cree conveniente que la evaluación consistiría en la revisión de los ejercicios ejecutados correctamente. Para evaluar el nivel ortográfico, se puede calcular el tanto por ciento de errores ortográficos, haciendo lo siguiente:

No de errores x 100

No de palabras escritas $=\%$ de faltas ortográficas.

\section{Conclusiones}

Un taller de lenguaje y comunicación como vía para mejorar la enseñanza aprendizaje de ortografía y comprensión lectora en estudiantes universitarios, debe fundamentarse desde varios aspectos como es el andragógico, pedagógico, psicológico y psicolinguiístico, entre otros, que son necesarios para la comprensión del lenguaje en sus funciones cognitivas y comunicativas.

El uso de talleres de trabajo, que desarrollen habilidades y competencias lingüísticas mediante la ejecución de tareas donde los estudiantes enfrenten situaciones comunicativas que los lleven a comprender y aplicar lo que aprenden como herramienta para resolver problemas educativos y profesionales.

El taller propone múltiples ejercicios para ser realizados individual y grupalmente en el que se debe ir superando etapas, que estimulen y potencien las capacidades lecto - lingüísticas. 


\section{Bibliografía}

Álvarez, Z. (1999). Didáctica, la escuela en la vida. La Habana: Editorial Pueblo y Educación, p. 09.

Cano, A. (2012). La metodología de taller en los procesos de educación popular. Buenos Aires, Argentina: Revista Latinoamericana de Metodología de las Ciencias Sociales vol. 02, no. 02 p. 22-52

Cassany, D. (2009). La lectura y la escritura en un mundo cambiante. Tokio: Seminario del Departamento de Español / Universidad de Sofía.

Dewey, J. (1938/1997). Experiencia \& Educación. Nueva York: Simón \& Schuster.

Duque, R. (1993). La evaluación en la ES Venezolana. Planiuc. Números 17-18, Aniversario X p 03

EGG. A. (1999). El taller: una alternativa de renovación pedagógica. Buenos Aires, Río de La Plata.

Fuentes, H. (1998). Dinámica del proceso docente educativo de la educación superior., Santiago de Cuba: Tesis (Ciencias Pedagógicas). Universidad de Oriente.

García, B. (1990). Saber expresarse y en- señar a expresarse. Informe de Investiga- ción. Cuba (Sancti Spiritus): Instituto Su- perior Pedagógico “Capitán Silverio Blanco Núñez.

García, E. (1975). Lengua y literatura. La Habana: Pueblo y Educación.

Goleman, D. (2000). La inteligencia emo- cional: Por qué es más importante que el cociente intelectual. México: Ediciones B.

Gutiérrez, N. (2013). Técnicas y estrategias de expresión escrita. Ilobasco: Compilado en la Dirección Departamental de Educación de Cabañas

León, D., Fernández, O. y Valdés, R. (2007). La actuación del profesor para el desarrollo de habilidades de expresión oral en estudiantes universitarios: resultados de una estrategia didáctica., Natal, Brasil: Revista Educação em Questão p. 8-20.

Morales, O., Cassany, D. (2008). Leer y escribir en la universidad: hacia la lectura y la escritura crítica de géneros científicos. Revista Memoralia, Universidad Nacional Experimental de los Llanos Ezequiel Zamora: p.4-10.

Ruiz, M. (2007). La Formación en competencias. Tres procesos metodológicos esenciales. Monterrey: Ed. Facultad de Filosofía y Letras, UANL. 
Rumeu, E. (2004). El enfoque cognitivo, comunicación y de orientación sociocultural. Dimensiones e indicadores de la competencia cognitiva, comunicativa y sociocultural. La Habana: Editorial Pueblo y Educación.

Rumeu, E. et al. (2006). El enfoque cognitivo, comunicativo y sociocultural en la enseñanza de la lengua y la literatura. La Habana: Editorial Pueblo y Educación.

Solé, I. (1998). Estrategias de lectura. Barcelona: Graó.

Tobón, S. (2006) Aspectos básicos de la formación basada en competencias. Bogotá: ECOE.

Yturralde, E. (2012). Andragogía. México: Worldwide. 
